University of Nebraska - Lincoln

DigitalCommons@University of Nebraska - Lincoln

Faculty Publications in Computer \& Electronics Electrical \& Computer Engineering, Department Engineering (to 2015)

$11-2006$

\title{
Multi-User UWB-IR Systems with Interleaved Coding-Modulation on Multipath Fading Channels
}

\author{
Michal M. Pietrzyk \\ Delft University of Technology \\ Keni Popovski \\ University of Wollongong \\ Tadeusz Wysocki \\ University of Nebraska-Lincoln, wysocki@uow.edu.au \\ Beata J. Wysocki \\ University of Wollongong \\ Jos H. Weber \\ Delft University of Technology
}

Follow this and additional works at: https://digitalcommons.unl.edu/computerelectronicfacpub

Part of the Computer Engineering Commons

Pietrzyk, Michal M.; Popovski, Keni; Wysocki, Tadeusz; Wysocki, Beata J.; and Weber, Jos H., "Multi-User UWB-IR Systems with Interleaved Coding-Modulation on Multipath Fading Channels" (2006). Faculty Publications in Computer \& Electronics Engineering (to 2015). 59.

https://digitalcommons.unl.edu/computerelectronicfacpub/59

This Article is brought to you for free and open access by the Electrical \& Computer Engineering, Department of at DigitalCommons@University of Nebraska - Lincoln. It has been accepted for inclusion in Faculty Publications in Computer \& Electronics Engineering (to 2015) by an authorized administrator of DigitalCommons@University of Nebraska - Lincoln. 


\title{
Multi-User UWB-IR Systems with Interleaved Coding-Modulation on Multipath Fading Channels
}

\author{
Michal M. Pietrzyk ${ }^{\dagger}$, Keni Popovski ${ }^{\ddagger}$, Tadeusz A. Wysocki ${ }^{\ddagger}$, Beata J. Wysocki ${ }^{\ddagger}$, and Jos H. Weber ${ }^{\dagger}$ \\ ${ }^{\dagger}$ Faculty of Electrical Engineering, Mathematics and Computer Science \\ Delft University of Technology \\ Mekelweg 4, 2628 CD Delft, The Netherlands \\ Email: $\{$ M.M.Pietrzyk, J.H.Weber\}@ewi.tudelft.nI \\ ${ }^{\ddagger}$ School of Electrical, Computer and Telecommunications Engineering \\ University of Wollongong \\ Northfields Ave, NSW 2522 Wollongong, Australia \\ Email: $\{$ Keni, Wysocki, BJW\}@uow. edu.au
}

\begin{abstract}
Interleaved coding-modulation (ICM) is a recently proposed method for ultra-wideband impulse radio (UWBIR) systems. ICM exploits the concept of chip interleaving, allowing to alleviate the problem of inter-symbol and inter-pulse interference commonly present in high data rate UWB-IR systems. In this paper, we extend previous work on ICM to scarcely populated multi-user scenarios. We propose a design of a deterministic chip interleaver based on time-hopping hyperbolic congruence sequences. We also review the main parameters of the random and hyperbolic interleavers. Our results indicate that the proposed type of interleaver yields similar performance to random interleavers but with the advantage of simpler implementation.
\end{abstract}

Keywords: Ultra-wideband, channel coding, multi-user system, inter-symbol interference, interleaving.

\section{INTRODUCTION}

One of the most promising candidates for future Wireless Local Area Networks (WLANs) and Personal Area Networks (PANs) is Ultra-Wideband (UWB) technology. A UWB system is defined as any communication system operating on a continuous frequency bandwidth larger than or equal to $500 \mathrm{MHz}$ [1]. UWB is characterized by distinctive properties that stem mainly from the utilization of the extremely wide bandwidth, i.e, support for a high date rate ( $>100 \mathrm{Mbps})$ transmission, immunity to multipath, and fine spatial and time resolution.

Since UWB communication systems are primarily designed for indoor applications, it is important to assure the coexistence of a number of transmitters in the covered area. In existing studies on multi-user interference (MUI) in UWB-IR systems, a Gaussian approximation (GA) is often used to model the interference stemming from the concurrent transmitters as an additive Gaussian process having uniform power spectrum over the bandwidth of interest [2], [3]. However, to be valid, the GA requires a large number of mutually independent random variables when no single variable or a group of variables can dominate. As a result, it gives inaccurate results in scarcely populated systems, in systems with a dominant interferer, and in systems operating on multipath fading channels [4]. For such cases the GA returns very optimistic results

Existing work on the modeling of the bit error rate (BER) performance assuming a non-Gaussian approach include the Quadrature rule approximation [5], characteristic function technique [6], [7] or Poisson distribution modeling [8]. In most of the papers, simple additive white Gaussian channels, perfect power control and bipolar pulse amplitude or pulse position modulation are considered. Another approach is taken in [9], where a method is provided to compute the conditional BER given some realizations of the channels. Although this technique is much simpler than the others, it is assumed that no inter-symbol interference (ISI) nor inter-pulse interference (IPI) is present.

In this paper, we characterize the BER performance of scarcely populated high data rate coded UWB-IR systems operating on the IEEE 802.15.3a multipath fading channels using a Poisson based analysis and Monte Carlo simulations. Effects of both types of interference, namely ISI and IPI, are considered. Furthermore, we investigate the performance of previously proposed interleaved coding-modulation [10] applied in combination with superorthogonal convolutional (SOC) coding or frame repetition (FR). Tests are conducted in a multi-user scenario focusing on the design of time-hopping (TH)-like sequences that are used for chip interleaving. Our results indicate that a deterministic interleaver based on hyperbolic congruence sequences may perform similarly to the random interleavers with the advantage of simple implementation. In our system model, the considered modulation is differential autocorrelation signalling with a single delay branch allowing for a simplified receiver architecture.

This paper is organized as follows. Section II presents the considered UWB-IR system model with particular insight into the principle of building the chip interleaver. Moreover, a detailed description of investigated sequences is provided. Section III focuses on the interleaver parameters characterization. In Section IV theoretical and simulation BER results are presented together with a discussion. Section V concludes the paper. 


\section{SYSTEM MODEL}

\section{A. UWB-IR Transmitter}

Generation of the transmitted signal waveform for one data packet is performed in the following way. A sequence of $N_{u}$ information bits $\mathbf{u}=\left(u_{0}, u_{1}, \ldots, u_{N_{u}-1}\right)$ is encoded according to the selected encoding function $\varphi$ resulting in a code bit sequence $\mathbf{d}=\varphi(\mathbf{u})=\left(d_{0}, d_{1}, \ldots, d_{N_{d}-1}\right)$, where $N_{d}$ denotes the number of code bits/modulation symbols in a packet. The considered modulation is of order one. A bijection $\varphi$ transforms a block of $k$ bits into a block of $n$ bits. In general, $\varphi(\mathbf{x})$ is a sequence obtained by partitioning the sequence $\mathbf{x}$ into subsequences of length $k$ and applying $\varphi$ on every subsequence. For a special case. when $k=n(=1)$, $\varphi(\mathbf{x})=\mathbf{x}$ and hence there is no channel coding. Code bits $d_{i}$ are fed into a differential autocorrelator $b_{i}=b_{i-1}\left(1-2 d_{i}\right)$. $\forall i \in\left\{0,1, \ldots, N_{d}-1\right\}$ which delivers a sequence of modulated symbols $\mathbf{b}=\left(b_{0}, b_{1}, \ldots, b_{N_{d}-1}\right)$, where $N_{d}$ can be calculated as $N_{d}=\frac{n}{k} N_{u}$.

The next operation is pulse grouping that results in a sequence $\tilde{\mathbf{s}}=\left(\tilde{s}_{0}, \tilde{s}_{1}, \ldots, \tilde{s}_{N-1}\right)$, where $\tilde{s}_{l}=b_{i}$, if $i N_{f} N_{p} \leq$ $l \leq i N_{f} N_{p}+N_{f}-1, \forall i \in\left\{0,1 \ldots N_{d}-1\right\}$, and $\tilde{s}_{l}=0$ otherwise. The number of frames per modulation symbol is $N_{f}$, whereas the number of chips per frame is denoted by $N_{p}$. The pulse grouping operation boils down to placing pulses at the beginning of every modulation symbol $b_{i}$. This insures orthogonality of the users' signals before passing them through the channel since chip interleaving will be operating on the same initial conditions for every user.

The following step is chip interleaving $\Pi_{p}: x \rightarrow \Pi_{p}(x)$, where the size of the interleaver is denoted by $L_{p}$. The mapping $\Pi_{p}$ is user-dependent. In general, a sequence $\Pi_{p}(\mathbf{x})$ is obtained by partitioning sequence $\mathbf{x}$ into subsequences of length $L_{p}$ and applying $\Pi_{p}$ on every subsequence. Here, $\Pi_{p}$ is applied on the sequence $\tilde{\mathbf{s}}$ yielding a sequence of interleaved chips $\tilde{\mathbf{v}}=\Pi_{p}(\tilde{\mathbf{s}})=\left(\tilde{v}_{0}, \tilde{v}_{1}, \ldots, \tilde{v}_{N-1}\right)$, where $N$ is the total number of chips in a data packet and can be calculated as $N=$ $N_{p} N_{f} N_{d}$. As compared with [10], polarity randomization is not considered here. The transmitted signal waveform for one data packet from user $i$ is thus given by

$$
s_{t}^{(\kappa)}(t)=\sum_{i=0}^{N-1} \tilde{r}_{i}^{(\kappa)} u\left(t-i T_{u}\right) .
$$

where $w(t)$ denotes the transmitted pulse of duration $T_{w}$.

\section{B. UWB-IR Receiver}

The received signal over a multipath fading channel in a $U$ multi-user system can be expressed as

$$
r^{\prime \prime}(t)=r^{\prime \prime(1)}(t)+I(t)+n(t),
$$

where $r^{\prime \prime(1)}(t)$ is the useful signal given by

$$
r^{\prime \prime(1)}(t)=A_{1} s_{t}^{(1)}(t) * g^{(1)}(t) * h^{(1)}(t)
$$

and $I(t)$ is the multi-user interference term expressed as

$$
I(t)=\sum_{\kappa=2}^{U} A_{\kappa} s_{t}^{(\kappa)}(t) * g^{(\kappa)}(t) * h^{(\kappa)}(t) .
$$

and $n(t)$ is additive white Gaussian noise. The channel impulse response of user $\kappa$ is denoted by $h^{(\kappa)}(t)$. In our system model, we assume perfect power control, i.e., the signal amplitudes satisfy $A_{1}=A_{2}=\ldots=A_{\kappa}=\ldots=A_{U}$. Furthermore, we assume that the impulse responses of the front-ends of every user's transceiver are the same, i.e., $g^{(1)}(t)=g^{(2)}(t)=\ldots=$ $g^{(\kappa)}(t)=\ldots=g^{(U)}(t)$.

The received sequence for one data packet may be expressed as $\mathbf{r}^{\prime \prime}=\left(r_{0}^{\prime \prime}, r_{1}^{\prime \prime} \ldots, r_{N-1}^{\prime \prime}\right)$, where $r_{i}^{\prime \prime}$ is the waveform $r^{\prime \prime}(t)$ on the interval $\left[i T_{w},(i+1) T_{w}\right]$. The first operation upon receiving the signal is chip deinterleaving, $\Pi_{p}^{-1}\left(\mathbf{r}^{\prime \prime}\right)$ yielding a sequence $\mathbf{z}=\Pi_{p}^{-1}\left(\mathbf{r}^{\prime \prime}\right)=\left(z_{0}, z_{1}, \ldots, z_{N-1}\right)$. Next, pulse de-grouping and time-filtering operations are performed resulting in the sequence $\breve{\mathbf{z}}=\left(\check{z}_{0}, \check{z}_{1}, \ldots, \check{z}_{N-1}\right)$, where $\breve{z}_{l}=z_{i N_{f} N_{p}+m} \delta_{m . c_{j}}$, $\forall 0 \leq l \leq N-1 . \forall 0 \leq i \leq N_{d}-1$, and $\forall 0 \leq m \leq$ $N_{p}-1$, where $\delta_{i, j}=1$ if $i=j$, and $\delta_{i, j}=0$ if $i \neq \bar{j}$. The pseudorandom assignment code used to distinguish the users and known at the receiver is $\mathbf{c}=\left(c_{0}, c_{1}, \ldots, c_{N_{f}-1}\right)$ with values $c_{i} \in\left\{0,1, \ldots, N_{p}-1\right\}, \forall i$. It is important to notice that the time-filtering operation boils down to selecting these chips from the sequence $\mathbf{z}$ that originally carried pulses at the transmitter side. The rest of the chips in the sequence $\mathbf{z}$ are zero padded. The result of deinterleaving is fed to the demodulator that correlates the signal with its symbol-delayed version as

$$
\hat{y}_{i}=\int_{i T_{*}}^{i T_{s}+T_{i}} \tilde{z}(t) \tilde{z}\left(t-T_{s}\right) d t .
$$

where $i$ is the symbol index and $\tilde{z}$ is a subsequence of $\overline{\mathbf{z}}$ with length $N_{f} N_{p}$ corresponding to one symbol of duration $T_{s}$. In our system setup, for the case of FR, a resulting sequence of real values written as $\hat{\mathbf{y}}=\left(\hat{y}_{0}, \hat{y}_{1}, \ldots, \hat{y}_{N_{d}-1}\right)$ is passed through a threshold detector yielding a sequence of binary values $\hat{\mathbf{y}}_{\mathrm{b}}=$ $[-1 \cdot \hat{\mathbf{y}}]$, where $[x]$ is equal to 1 for $x>0$ and 0 for $x \leq 0$. For the case of SOC coding, the sequence of real values $\hat{\mathbf{y}}$ is fed directly to the soft-input Viterbi decoder. In general, the sequence $\hat{\mathbf{y}}_{\mathbf{b}}$ is decoded with a decoding function $\psi\left(\hat{\mathbf{y}}_{\mathbf{b}}\right)$ yielding a decoded bit sequence $\hat{\mathbf{u}}=\left(\hat{u}_{0}, \hat{u}_{1}, \ldots, \hat{u}_{N_{u}-1}\right)$.

\section{SOC Coding and FR}

Two particular cases of the ICM scheme are investigated, one further referred to as a UWB-IR system with SOC coding, for which $k=1, n>1$, and $N_{f}=1$, and the second, further referred to as a UWB-IR system with frame repetition, in which there is no coding scheme applied. i.e., $k=n=1$ and $N_{f}>1$. In order to allow a fair comparison between the two schemes, we ensure an equal number of transmitted pulses per information bit by setting the length of the SOC code, $n$, equal to the number of frames per symbol of the FR scheme, $N_{f}$.

\section{UWB Channel Model}

The considered IEEE 802.15.3a UWB channel model is a modified Saleh-Velenzuela $(\mathrm{S}-\mathrm{V})$ model. In this paper, we refer to the channel scenario CM3 that represents a non-line-ofsight transmission with distances between the transmitter and the receiver ranging from 4 to $10 \mathrm{~m}$. For information on the channel description, the reader is requested to study [11]. 


\section{E. Interleaver Sequence Design}

Orthogonal sequencing is employed in the design of the chip interleaver whose purpose is to intersperse the pulses corresponding to all users for all the symbols transmitted. A unique sequence indicating the reordering mapping of the chips periodically repeated for the whole data packet is assigned to each user. However, unlike in conventional timehopping applications, pulses dispersed through interleaving are generally not in time consecutive order. This introduces time diversity allowing for spreading of bursty errors caused by fading over several code words. Both time and frequency hopping codes may be used for this application, provided that they adhere to the following constraints:

- Each sequence consists of unique values required for the interleaver's one-to-one mapping operation.

- High level of orthogonality between the sequences is ensured to avoid catastrophic collisions and minimize the multi-access interference.

For the purpose of our analysis, we have chosen hyperbolic congruence and random sequences. The former are known to have very good auto- and cross-correlation properties [12] and a defined structure that can be easily implemented in the system. Interleavers based on the latter, however, provide good BER performance but lack of compact representation.

Hyperbolic congruence sequences: The placement operator is defined as [13]

$$
\Upsilon(i)=\frac{a}{i}\left(\bmod p^{\prime}\right), \quad 1 \leq a, i \leq p^{\prime}-1,
$$

where $p^{\prime}$ is a prime number, $a$ is the sequence index, and $i$ is the sequence element index. The cardinality of the set of sequences equals $p^{\prime}-1.1 / i$ is the unique inverse of $i$ in the Galois field GF(p') and can be calculated as [14]

$$
\frac{1}{i}=i^{p^{\prime}-2}\left(\bmod p^{\prime}\right) \text {. }
$$

\section{INTERLEAVER PROPERTIES}

\section{A. Delays and Latency}

The delay function of an interleaver, $\Delta(i)$, is defined as

$$
\Delta(i)=i-\Pi(i), \quad \forall i \in \mathbf{Z}, \mathbf{Z}=\left\{1,2, \ldots, L_{p}\right\} .
$$

The interleaver operation can thus be described as

$$
y(i)=x(i-\Delta(i)) .
$$

An example of operation of an interleaver is shown in Table I. The minimum delay is defined as

$$
\Delta_{\min }=\inf _{i \in \mathbf{Z}} \Delta(i)
$$

whereas the maximum delay is

$$
\Delta_{\max }=\sup _{i \in \mathbf{Z}} \Delta(i) .
$$

The characteristic delay is given by

$$
\tilde{\Delta}=\Delta_{\max }-\Delta_{\min } \text {. }
$$

\section{B. Dispersion}

The randomness of an interleaver can be measured by the dispersion parameter. The normalized dispersion is given by [15]

$$
\begin{array}{r}
\gamma=\mid\left\{\left(\Delta_{x}, \Delta_{y}\right) \mid \Delta_{x}=j-i, \Delta_{y}=\Pi(j)-\Pi(i),\right. \\
\left.1 \leq i<j \leq L_{p}\right\} \mid\left(L_{p}\left(L_{p}-1\right) / 2\right),
\end{array}
$$

where the cardinality of a set is denoted by $|\cdot|$. A low value of dispersion means that the interleaver is very regular. The smallest value of the dispersion is achieved by the identity permutation.

\section{Spread}

The spread of an interleaver is a measure of the distance between interleaved symbols that were "close" to each other before interleaving. The design of an interleaver characterized by the square spread parameter $S$ guarantees that if two input symbols to the interleaver $\Pi$ are within a distance $S_{1}$, they cannot be mapped in such a way that a distance between the output symbols is less than $S_{2}$, and usually $S_{1}=S_{2}=S$ [16]. Thus considering two indices $i$ and $j$ such that

$$
0<|i-j| \leq S
$$

the design imposes that

$$
|\Pi(i)-\Pi(j)| \geq S .
$$

Spread parameters of the interleavers considered in this paper equal 1.

\section{Input-Output Separation Spectrum}

Fig. 1 shows the input-output separation spectra (IOSS) of a random and hyperbolic interleaver, respectively. This graph represents two-dimensional histograms with the axes being the separation for all possible element-pairs at the input and output of the interleaver. The IOSS indicates the level of randomness of an interleaver since the fewer the possible combinations of the element-pairs, the more structured the interleaver is. As can be observed from Fig. 1, the IOSS of the two considered interleavers look similar, however, with slight superiority with respect to the randomness level of the random interleaver. The parameters of interleavers under examination averaged over five realizations are gathered in Table II. We observe small differences in values of the delay functions. Furthermore, the value of the dispersion of a random interleaver is larger indicating the higher level of randomness of this type of interleaver. This fact is also visible when comparing the IOSS of a random and hyperbolic interleaver in Fig. 1.

\section{Performance Evaluation ANd Discussion}

\section{A. Theoretical Analysis}

In this section, we derive a bound on the BER for multiuser UWB-IR systems when the effects of the AWGN are neglected ( $E_{b} / N_{0}$ tends to infinity) and the only source of errors are pulse collisions due to simultaneous transmission of signals corresponding to different users. In the following, we 
TABLE I

EXAMPLE OF OPERATION OF AN INTERLEAVER

\begin{tabular}{|c|c|c|c|c|c|c|c|c|}
\hline $\mathrm{i}$ & 1 & 2 & 3 & 4 & 5 & 6 & $\ldots$ & 400 \\
\hline \hline$\Pi(i)$ & 5 & 1 & 8 & 10 & 3 & 2 & $\ldots$ & 100 \\
\hline$\Delta(i)$ & -4 & 1 & -5 & -6 & 2 & 4 & $\ldots$ & 300 \\
\hline
\end{tabular}

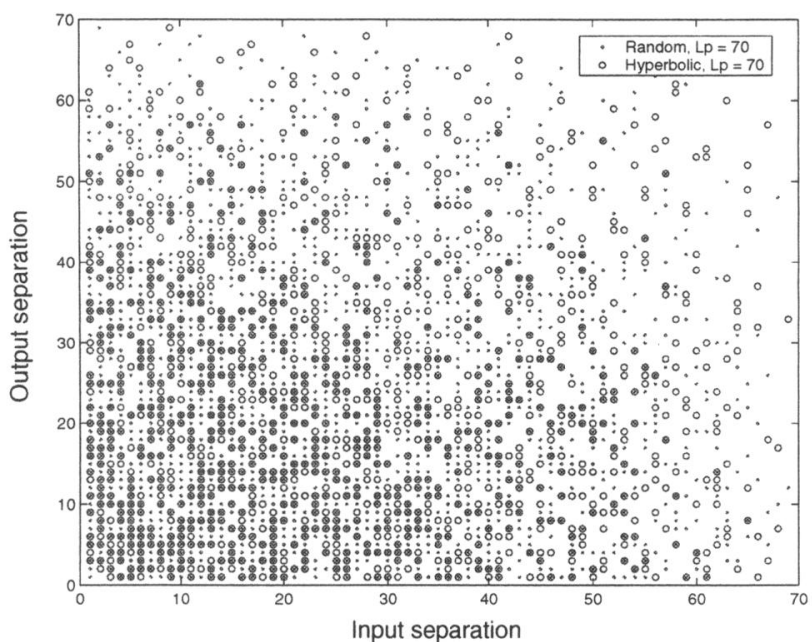

Fig. 1. The input-output separation spectrum of a random interleaver. The input separation is $|i-j|$, whereas the output separation is $|\Pi(i)-\Pi(j)|$. For clarity of the figure, the length of the interleaver equals $L_{p}=70$.

assume that the channel model is a binary symmetric channel (BSC) with the crossover probability $p$ and that hard decision decoding is being performed at the receiver. We also assume that a pulse inter-arrival process is a Poisson process. Such an assumption allows as to relate the pulse collisions probability to the ALOHA collision probability, when the information unit is the pulse instead of the packet.

The probability that there are exactly $\rho$ occurrences in a Poisson process is

$$
P(X=\rho)=\frac{e^{-\lambda} \lambda^{\rho}}{\rho !},
$$

where $\lambda$ is a positive real number equal to the expected number of occurrences that take place during a given interval. For the case of zero collisions, $\rho=0$, one may write

$$
P(X=0)=e^{-\lambda} \text {. }
$$

However, since a vulnerable interval equals to two time windows, which corresponds to the unslotted ALOHA case, the probability of a successful transmission is

$$
P_{1}=e^{-2 \lambda} \text {. }
$$

The pulse arrivals in a multi-user scenario are modeled by a Poisson process with the pulse arrival rate $\lambda$ that equals to

$$
\lambda=\lambda^{\prime}(U-1),
$$

where $\lambda^{\prime}$ is the pulse arrival rate for a single user UWB-IR system and $\lambda^{\prime}=T_{w}^{\prime} / T_{f}$ with $T_{w}^{\prime}$ denoting the effective pulse
TABLE II

INTERLEAVER PARAMETERS

\begin{tabular}{|c|c|c|c|c|c|c|}
\hline Interleaver & $L_{p}$ & $\overline{\Delta_{\min }}$ & $\overline{\Delta_{\max }}$ & $\overline{\bar{\Delta}}$ & $\bar{S}$ & $\bar{\gamma}$ \\
\hline \hline Random & 400 & -372.4 & 384.6 & 757 & 1 & 0.81 \\
\hline Hyperbolic & 400 & -367.8 & 382.8 & 750.6 & 1 & 0.65 \\
\hline
\end{tabular}

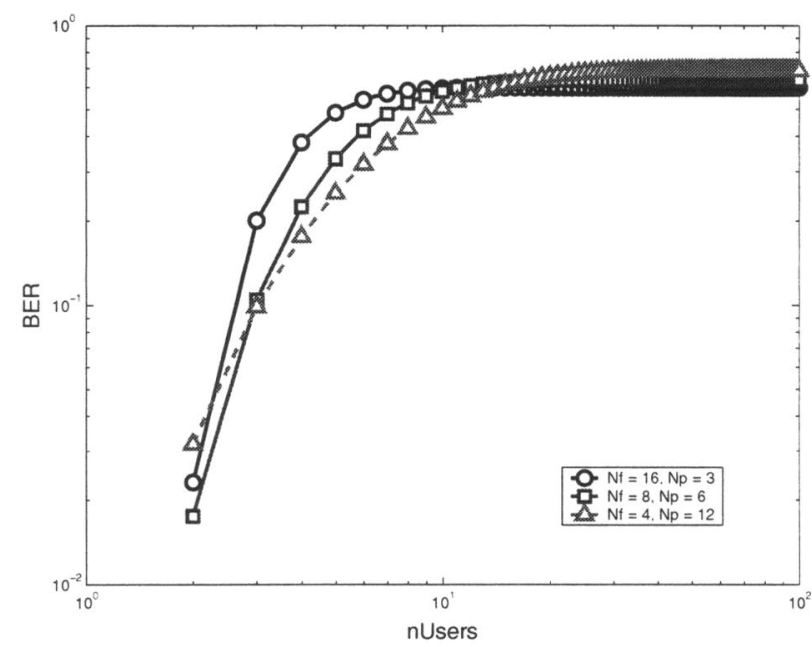

Fig. 2. Bounds on the BER of UWB-IR systems with FR and without ICM for different system parameter settings $N_{f}$ and $N_{p}$. We set $T_{w}^{\prime}=T_{w}=0.167 \mathrm{~ns}$ that allows to capture $100 \%$ of the pulse energy.

duration in which the most pulse energy is concentrated and $T_{f}=N_{p} T_{w}$ being the mean time between the pulses (frame time). We can write that

$$
\lambda=\frac{T_{w}^{\prime}}{T_{f}}(U-1) .
$$

The resulting pulse collision probability can be expressed as

$$
P_{\text {col }}=1-P_{1}=1-e^{-2(U-1) \frac{T_{u}^{\prime}}{T_{f}}} .
$$

The crossover probability is

$$
p=\frac{P_{\text {col }}}{2},
$$

where a division by two is performed to account for constructive effects of the pulse collisions.

1) Frame Repetition: In our UWB-IR system model an information bit / modulation symbol is represented by $N_{f}$ frames each containing one pulse. Assuming that an information bit error occurs when more than or equal to $N_{f} / 2$ pulses are in error, the bit error probability is given by

$$
P_{b}=\sum_{\left\lceil i=\frac{N_{f}}{2}\right\rceil}^{N_{f}}\left(\begin{array}{c}
N_{f} \\
i
\end{array}\right) p^{i}(1-p)^{N_{f}-i},
$$

where the $\lceil\cdot\rceil$ denotes a rounding towards infinity operation.

Fig. 2 shows calculated bounds on the BER for different configurations of UWB-IR system parameters, i.e., $N_{f}$ and 


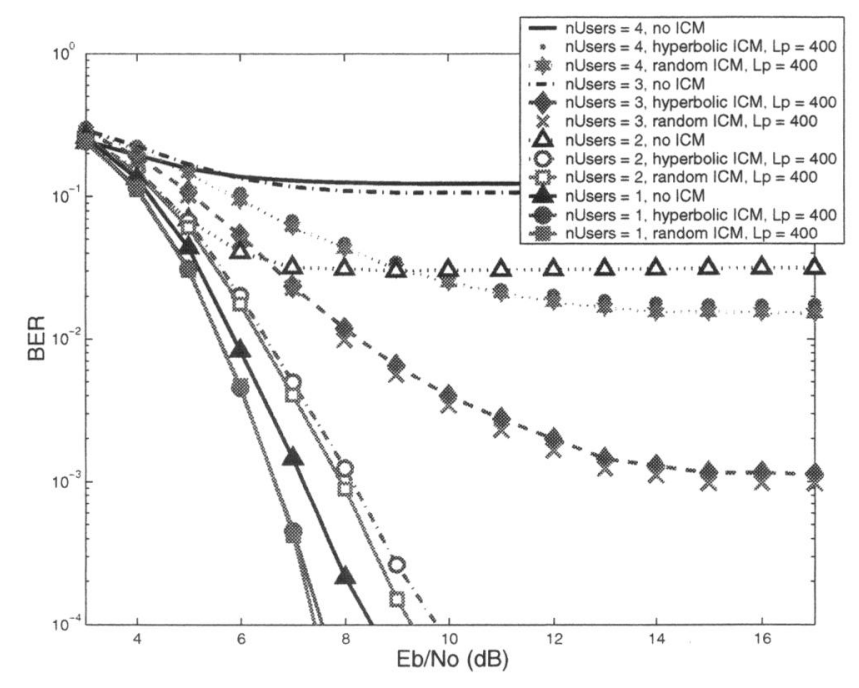

Fig. 3. The bit error rate of UWB-IR systems with SOC coding and with or without ICM. Two types of length $L_{p}=400$ sequences considered, namely hyperbolic and random.

$N_{p}$ when frame repetition is applied. The data rates for the considered parameter settings are the same. We observe that for a two-user scenario, the best performance is achieved when a single information bit is represented by 8 pulses with 5 empty-chips-long separation $\left(N_{p}=6\right)$, whereas for higher number of active users, i.e. when $U>3$, lowering the number of pulses and at the same time increasing the number of empty chips yields better BER results. Explanation of this phenomenon lies in the fact that increasing the number of empty chips and thus time separation between the pulses lowers the pulse collision probability, since more pulses can be placed within the frame without causing any collisions. On the other hand, when $U<3$, it is better to insure the correction capability introduced by a reasonable number of frames (here $N_{f}=8$ ), so that the possible errors caused by pulse collisions can be eliminated. Interestingly, the BER curves do not approach 1 nor 0.5 when $U \rightarrow \infty$, although the crossover probability $p$ tends to 0.5 value. It is due to the fact that when $p \rightarrow 0.5$ and is plugged into (23), then depending on $N_{f}, P_{b} \rightarrow 0.6875,0.63672$ or 0.59819 , for $N_{f}=4,8$, and 16 , respectively.

2) SOC Coding: The bit error rate performance may be bounded by the known union upper bound [17]. However, due to obtained large values of the crossover probability, $p>0.1$ for $U>1$, the bound becomes unacceptably loose. A bit error rate analysis based on this bound might be appropriate when the data rate of the considered UWB-IR systems is much smaller or/and channel conditions are better (e.g. no multipath fading, ISI, nor IPI present) so that the crossover probability is much smaller than 0.1 .

\section{B. Simulation Results}

The performance of scarcely populated high data rate (125Mbps) UWB-IR systems is evaluated through Monte

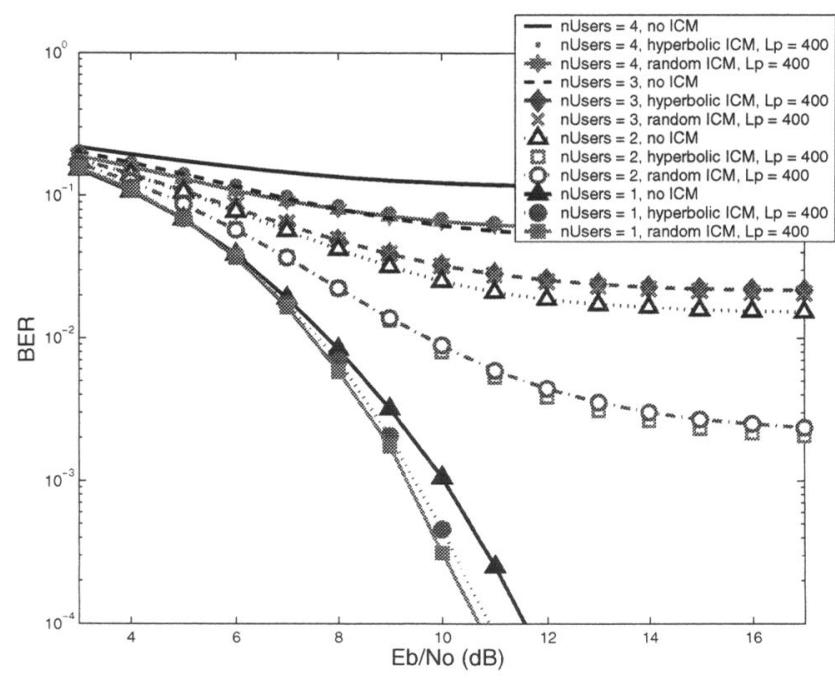

Fig. 4. The bit error rate of UWB-IR systems with FR and with or without ICM. Two types of length $L_{p}=400$ sequences considered, namely hyperbolic and random.

Carlo simulations with 40 independent channel realizations and 2000 information bits in every packet. Our assumption is that the channel is stationary during transmission of one data packet. For every channel realization a unique sequence is assigned to the intended user in order to eliminate performance dependence on the selected sequences and average the results. For interferers, the value of $a$ determining the sequence is pseudorandomly chosen from the set $\{1,2, \ldots, U-1\}$. In order to model a situation that takes place often in practice that none of the users starts its transmission at the same time as the others, a random circular shift is added to the sequences. Perfect power control is assumed, i.e., powers of the signals corresponding to every active user are equal. The data rates of the considered synchronous UWB-IR systems are identical and the bandwidth expansions introduced by SOC coding and FR are equal.

Fig. 3 and 4 show the BER performance of scarcely populated UWB-IR systems with and without the ICM technique when the total number of users $U=1,2,3$, or 4 . As can be seen from both figures, the best BER performance is achieved when there is only one active user in the system. Increasing the number of active users worsens the BER. For $U>1$, when $E_{b} / N_{0}$ values are high, Fig. 3 and 4 reveal an error floor indicating that the performance is determined by the MUI, ISI, and IPI types of interference rather than the noise. The only exception is an $U=2$ multi-user UWB-IR system with ICM and SOC coding applied, for which, for the BER range as in the figures, we do not observe the error floor. For the parameter setting as in our system model, i.e., the number of chips per frame, $N_{p}=6$, the length of the SOC code, $n=8$, and the number of frames per symbol, $N_{f}=8$, the maximal number of users is 6 . However, when considering target BER values lower than $10^{-2}$, we notice that the maximal number of users served by the UWB-IR system 


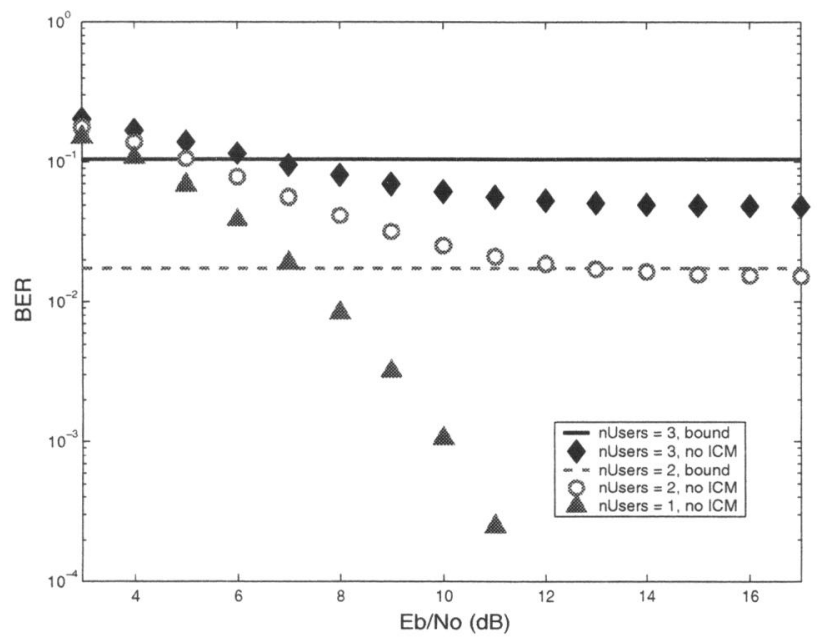

Fig. 5. The bit error rate of UWB-IR systems with FR and without ICM bounds and simulation results.

with the ICM technique and SOC coding or FR applied is 3 or 2 , respectively. This observation may suggest that for multiuser scenarios with high data rate transmissions ( $>100 \mathrm{Mbps}$ ) over multipath fading channels, channel codes much stronger than SOC are required, especially for UWB-IR systems based on a single delay branch differential autocorrelation receiver. As illustrated in both figures, for a single user scenario, the coding gain introduced by the ICM technique is about $1 \mathrm{~dB}$ at the $10^{-4}$ BER level. When $U>1$, we observe larger coding gain, however, at the higher BER values. The time-filtering operation performed at the receiver introduces a significant improvement in the BER performance that may be visible when comparing Fig. 3 and 4 with Fig. 4 in [10]. For both methods of protection against errors, i.e., SOC coding and FR, hyperbolic and random chip interleaving yield similar BER performance. This phenomenon has its roots in similar values of interleaver parameters. Moreover, for both interleaver types, the most important parameter, i.e., spread equals $S=1$. It is imperative to point out here that interleavers based on hyperbolic congruence sequences do not have major drawbacks of random interleavers, i.e., lack of compact representation that could lead to simple implementation and lack of adequate analysis. Fig. 5 presents a comparison of the derived bound on the BER and actual BER performance obtained from simulations for the case of no ICM technique applied. When the number of active users $U=2$, the bound provides a good approximation of the BER. However, for $U=3$ and high values of the BER, the bound becomes loose, which can be noticed as an offset that is due to the inaccuracy of the modeling of MUI by the Poisson process.

\section{Closing Comments}

In this paper, we have evaluated the BER performance of scarcely populated high data rate coded UWB-IR systems operating on multipath fading channels. We have proposed a design of a deterministic chip interleaver for the ICM technique based on hyperbolic congruence sequences. We have also reviewed the main parameters of the random and hyperbolic interleavers. Our results indicate that the proposed type of the interleaver yields similar performance to the random interleavers but with the advantage of simpler implementation. We have also found that high data rate UWB-IR systems based on a single delay branch differential autocorrelation receiver perform poor in multipath fading channels, allowing, for the target BER levels lower than $10^{-2}$, to serve up to 3 or 2 active users for SOC coding or FR, respectively. The presented Poisson-based theoretical analysis may be helpful in a rough approximation of the BER performance of UWB-IR systems operating on correlated channels (when no chip interleaving is applied). For such cases, the Poisson assumption exemplifies the worst case scenario.

\section{REFERENCES}

[1] FCC, "Revision of part 15 of the commission's rules regarding ultrawideband transmission systems," First Report and Order, ET Docket, FCC 02-8, pp. 98-153, Feb./Apr. 2002.

[2] M. Z. Win and R. A. Scholtz. "Ultra-wide bandwidth time-hopping spread spectrum impulse radio for wireless multiple-access communications," IEEE Trans. Commun., vol. 48, no. 4, pp. 679-691, Apr. 2000.

[3] F. Ramirez-Mireles, "Performance of ultra-wideband SSMA using timehopping and M-ary PPM," IEEE J. Select. Areas Commun., vol. 19, no. 6, pp. 1186-1196, June 2001.

[4] M. O. Sunay and P. J. McLane, "Calculating error probabilities for DS CDMA systems: when not to use the Gaussian approximation," in Proc. IEEE GLOBECOM'96, vol. 3, Nov. 1996, pp. 1744-1748.

[5] G. Durisi and S. Benedetto, "Performance evaluation of TH-PPM UWB systems in the presence of multiuser interference," IEEE Commun. Lett., vol. 7, no. 5, pp. 224-226, May 2003.

[6] B. Hu and N. Beaulieu, "Exact bit error rate analysis of TH-PPM UWB systems in the presence of multiple-access interference," IEEE Commun. Lett. vol. 7, no. 12, pp. 572-574. Dec. 2003.

[7] M. Sabattini, E. Masry, and N. Beaulieu, "A non-gaussian approach to the performance analysis of UWB TH-BPPM systems," in Proc. IEEE UWBST'03, Nov. 2003, pp. 52-55.

[8] C. Bi and J. Hui, "Multiple access capacity for ultra-wideband radio with multi-antenna receivers," in Proc. IEEE UWBST'(02, May 2002, pp. 151-155.

[9] R. Merz and J.-Y. Boudec, "Conditional bit error rate for an Impulse Radio UWB channel with interfering users," in Proc: IEEE Int. Conf. on Ultra-Wideband (ICU'05), Sept. 2005, pp. 130-135.

[10] M. M. Pietrzyk and J. H. Weber, "Performance of UWB-IR with polarity randomization and interleaved coding-modulation on multipath fading channels," in Proc. IEEE Vehicular Technology Conf. (VTC'05), May 2005 .

[11] R. J. Foerster, "Channel modeling sub-committee report (final)," Tech. Rep. P8()2.15-()2/49()r1-SG3a. IEEE P8(12.15 Working Group for Wireless Personal Area Networks (WPANs). Feb. 2003.

[12] O. Moreno and S. V. Maric. "A new family of frequency-hop codes," IEEE Trans. Commun., vol. 48, no. 8, pp. 1241-1244, Aug. 2000.

[13] S. V. Maric and E. L. Titlebaum. "A class of frequency hop codes with nearly ideal characteristics for use in multiple-access spread-spectrum communications and radar and sonar systems," IEEE Trans. Commun., vol. 40, no. 9, pp. 1442-1447, Sept. 1992.

[14] A. V. Jovancevic and E. L. Titlebaum, "New coding schemes for increased number of users or messages in frequency-hopped multilevel FSK," in Proc. IEEE VTC'96, vol. 3, Apr. 1996, pp. 1732-1735.

[15] O. Y. Takeshita and D. J. Costello, "New deterministic interleaver designs for turbo codes," IEEE Trans. Inform. Theory, vol. 46, no. 6 , pp. 1988-2006, Sept. 2000.

[16] L. Dinoi and S. Benedetto, "Variable-size interleaver design for paralell turbo decoder architectures," IEEE Trans. Commun., vol. 53, no. 11, pp. 1833-1840, Nov. 2005.

[17] J. G. Proakis, Digital Communications. Singapore: McGraw-Hill Companies Inc., 2001 